\title{
Peculiar Features of Entangled States with Post-Selection
}

\author{
Yakir Aharonov ${ }^{1,2}$, Shmuel Nussinov ${ }^{1,2}$, Sandu Popescu ${ }^{3}$, and Lev Vaidman ${ }^{1,2}$ \\ 1 School of Physics and Astronomy, Tel Aviv University, Tel Aviv, Israel \\ 2 Institute for Quantum Studies, Chapman University, 1 University Dr, Orange, CA, USA and \\ 3 H.H.Wills Physics Laboratory, University of Bristol, Tyndall Avenue, Bristol BS8 1TL, U.K.
}

(Dated: September 2, 2018)

\begin{abstract}
We consider quantum systems in entangled states post-selected in non-entangled states. Such systems exhibit unusual behavior, in particular when weak measurements are performed at intermediate times.
\end{abstract}

PACS numbers: 03.65.Ta

Measurements performed on pre- and post-selected quantum systems often exhibit peculiar results. One particular example is that of a single particle which is found with certainty in any one of a large number of boxes if only this box is opened 1, 2]. In spite of experimental implementations of these ideas $[3-5]$, there is still some controversy about this example [6 20]. Even more surprising is the fact that outcomes of weak measurements 21], namely, standard von Neumann measurements with weakened interaction yield weak values which might be far away from the range of possible eigenvalues. This feature led to practical applications for high precision measurements [22, 23]. Here we consider the peculiar features of quantum systems when the pre-selected state is entangled.

First, let us consider the case when the pre-selected state is the following Greeberger-Horne-Zeilinger (GHZ)like entangled state of $N$ spin- $\frac{1}{2}$ particles:

$$
|\Psi\rangle_{1}=\frac{1}{\sqrt{2}}\left(\prod_{n=1}^{N}\left|\uparrow_{z}\right\rangle_{n}+\prod_{n=1}^{N}\left|\downarrow_{z}\right\rangle_{n}\right) .
$$

Just like the original three-particle GHZ state, it can be used to show that no local hidden variable theory is consistent with the predictions of quantum theory.

To this end consider the following sets of measurements. The first set consists of $\sigma_{x}$ measurements performed over all particles. The second set consists of $\sigma_{x}$ measurements for all particles except for two particles for which $\sigma_{y}$ are measured. Since

$$
\prod_{n=1}^{N} \sigma_{x}^{(n)}|\Psi\rangle_{1}=|\Psi\rangle_{1},
$$

and

$$
\prod_{n \neq k, l}^{N} \sigma_{x}^{(n)} \sigma_{y}^{(k)} \sigma_{y}^{(l)}|\Psi\rangle_{1}=-|\Psi\rangle_{1}
$$

the outcomes of the two sets of measurements should fulfill:

$$
\prod_{n=1}^{N} \sigma_{x}^{(n)}=1
$$

and

$$
\prod_{n \neq k, l}^{N} \sigma_{x}^{(n)} \sigma_{y}^{(k)} \sigma_{y}^{(l)}=-1
$$

respectively.

Next consider three particular cases of equation (5) where the pairs of particles for which $\sigma_{y}$ was measured were chosen from a triplet of particles $s, t$, and $r$.

We then have

$$
\begin{array}{r}
\prod_{n=1}^{N} \sigma_{x}^{(n)}=1, \\
\prod_{n \neq s, t}^{N} \sigma_{x}^{(n)} \sigma_{y}^{(s)} \sigma_{y}^{(t)}=-1, \\
\prod_{n \neq s, r}^{N} \sigma_{x}^{(n)} \sigma_{y}^{(s)} \sigma_{y}^{(r)}=-1, \\
\prod_{n \neq t, r}^{N} \sigma_{x}^{(n)} \sigma_{y}^{(t)} \sigma_{y}^{(r)}=-1 .
\end{array}
$$

Had there been local hidden variables, then all variables should have values, i.e., prior to the measurement every particle should "know" the value of all spin components and, in particular, the $x$ and the $y$ components. These values should fulfill all four equations (6). Just like in the original GHZ case, this is impossible since the product of the left hand sides is the product of squares (or fourth powers) of the spin variables and the product of the right hand sides is -1 .

In order to see surprising results we will consider the particular post-selected state $|\Psi\rangle_{2}$ in which all spins were found with $\sigma_{x}=1$, i.e.,

$$
|\Psi\rangle_{2}=\prod_{n=1}^{N}\left|\uparrow_{x}\right\rangle_{n} .
$$

A unique feature of this particular pre- and post-selection is that at intermediate times no pair of particles can be found with the same $\sigma_{y}$, otherwise, the requirement (5) cannot be fulfilled. 
To see this in a more dramatic way we map states of $N$ spin- $\frac{1}{2}$ particles into the states of $N$ distinguishable particles which can reside in two spatially separated boxes $A$ and $B$ :

$$
\left|\uparrow_{y}\right\rangle \equiv|A\rangle, \quad\left|\downarrow_{y}\right\rangle \equiv|B\rangle,
$$

where $|A\rangle(|B\rangle)$ is the state of the particle in box $A(B)$. Thus, we have $N$ (which might be as large as we want) particles in two boxes, but if we try to find any specific pair of particles in one box we are bound to fail! This is a very paradoxical situation for $N \geq 3$. The probability of the post-selection which leads to this situation is $\frac{1}{2^{N}}$, but for $N$ which is not too large it is feasible to implement experimentally.

The "paradox" in this example follows from i) the obvious classical observation that when three or more particles are located in two boxes, then at least one pair has to be in one box and ii) the quantum property which follows from the joint pre-and post-selection (but not from pre- or post-selection separately) that no pair of particles can be found in a single box.

Hardy has already proposed 24] a related experiment on a pre- and post-selected system. It can be viewed as an experiment with two particles in two boxes [25]. The particles were prepared in the entangled state

$$
\left|\Psi_{1}\right\rangle=\frac{1}{\sqrt{3}}\left(|A\rangle_{1}|B\rangle_{2}+|B\rangle_{1}|A\rangle_{2}+|B\rangle_{1}|B\rangle_{2}\right) .
$$

The preparation started with the non-entangled state

$$
|\Psi\rangle=\frac{1}{2}\left(|A\rangle_{1}+|B\rangle_{1}\right)\left(|A\rangle_{2}+|B\rangle_{2}\right),
$$

and entanglement was achieved by projecting out the state $|A\rangle_{1}|A\rangle_{2}$. Then, it was post-selected in the state

$$
\left|\Psi_{2}\right\rangle=\frac{1}{2}\left(|A\rangle_{1}-|B\rangle_{1}\right)\left(|A\rangle_{2}-|B\rangle_{2}\right) .
$$

The paradoxical feature here is that at intermediate times each particle is found in box $A$ if it is searched for there, but the two particles cannot be found together in $A$. The fact that each particle is (in this particular sense) in $A$ follows from the joint pre- and post-selection, while the fact that both are not there, follows directly from the pre-selection.

A generalization of this for the case of $N$ particles is the pre-selection of the state

$\left|\Psi_{1}\right\rangle=\frac{1}{\sqrt{N^{2}-N+1}}\left[(N-1) \prod_{n=1}^{N}|B\rangle_{n}+\sum_{n=1}^{N}|A\rangle_{n} \prod_{j \neq n}^{N}|B\rangle_{j}\right]$,

and the post- selection of the state

$$
\left|\Psi_{2}\right\rangle=\frac{1}{\sqrt{2^{N}}} \prod_{n=1}^{N}\left(|A\rangle_{n}-|B\rangle_{n}\right) .
$$

Every one of the $N$ particles is found with unit probability in box $A$ if it is searched for there. However, not only that they cannot all be found in $A$, any number of particles larger than one cannot be found there.

All these peculiarities of pre- and post-selected systems can be seen, albeit in a much more complicated way, in the standard formalism of quantum mechanics with a single quantum state evolving from the past to the future. The most surprising features of pre- and post-selected quantum systems are manifested in the outcomes of weak measurements [21]. Any standard measuring procedure with weak enough coupling performed on a quantum system pre-selected in state $|\Psi\rangle_{1}$ and post-selected in state $|\Psi\rangle_{2}$ yields, instead of one of the eigen values of the measured observable $O$, the weak value of $O$ given by the following expression:

$$
O_{w} \equiv \frac{\left\langle\Psi_{2}|O| \Psi_{1}\right\rangle}{\left\langle\Psi_{2} \mid \Psi_{1}\right\rangle}
$$

The weak value might be far away from the eigenvalues of $O$ and the standard quantum formalism can explain the outcome by surprising universal interference phenomenon of the pointer of the measuring device.

The following two theorems connect weak and "strong" (standard von Neumann) measurements [1]: i) if, for a pre- and post-selected system the outcome $o_{i}$ of the strong measurement of $O$ is known with certainty, then the weak value is the same $O_{w}=o_{i}$; ii) if, for a pre- and post-selected system the weak value of a dichotomic variable $O$ is equal to one of the eigenvalues $o_{i}$, then a strong measurement of $O$ will yield this value $o_{i}$ with certainty.

In the above generalized examples with $N$ particles, weak measurements suggest that, in some sense, all particles are in box $A$ in spite of the fact that at most one particle can be found in $A$. Indeed, the additive property of weak values implies:

$$
\left(\sum_{n=1}^{N} \mathbf{P}_{\mathbf{A}}^{(n)}\right)_{w}=\sum_{n=1}^{N}\left(\mathbf{P}_{\mathbf{A}}^{(n)}\right)_{w}=N .
$$

Note, however that the weak measurement of the product of the projection operators vanishes,

$$
\left(\prod_{n=1}^{N} \mathbf{P}_{\mathbf{A}}^{(n)}\right)_{w}=0\left[\neq \prod_{n=1}^{N}\left(\mathbf{P}_{\mathbf{A}}^{(n)}\right)_{w}\right]
$$

because the strong value of the product vanishes with certainty.

Let us return to the example with $N$ pre- and postselected particles in two boxes in which every particular pair of particles cannot be found in the same box. It is instructive to consider this example for identical spinless bosons. The statement that strong measurement cannot find any particular pair of particles in any single box is then meaningless because there is no "particular pair" among $N$ identical particles. Nevertheless, we still can make a statement about weak measurements. Let us assume that when two particles are in the same box, there is a potential $V$ between them. Weak measurement of 
the interaction energy between $N$ particles in the two boxes in our pre- and post-selected state should then yield zero! This can be seen in a straightforward way by calculating the weak value of the potential energy $\sum_{n \neq m} V \mathbf{P}_{\mathbf{A}}^{(n)} \mathbf{P}_{\mathbf{B}}^{(m)}$ for a system pre-selected in state (11) and post-selected in state (7). According to the mapping (8) the particles are described (up to normalization) by the following two-state vector

$$
\prod_{n=1}^{N}\left(\left\langle\left.A\right|_{n}+\left\langle\left. B\right|_{n}\right)\left(\prod_{n=1}^{N}\left(|A\rangle_{n}-i|B\rangle_{n}\right)+\prod_{n=1}^{N}\left(|B\rangle_{n}-i|A\rangle_{n}\right)\right)\right.\right. \text {. }
$$

Some intuition and an alternative proof of this result is as follows. The potential energy is the same for identical and nonidentical particles. For nonidentical particles, the measurement of the potential energy of any specific pair yields zero for this particular pre- and postselection. Therefore, the weak value of the potential energy for this pair is zero. Weak values are additive: $(A+B)_{w}=A_{w}+B_{w}$. Thus, the weak value of the potential energy of all pairs together is zero. Therefore, the weak value of the potential energy of $N$ identical particles is also zero.

Our next example is based on the most famous entangled state, the Einstein-Podolsky-Rosen-Bohm state (EPR), which is a singlet state of two spin- $\frac{1}{2}$ particles. It has been noted 26] that this pre-selected state when post-selected with a particular product state has unusual property at intermediate times. The two state vector is:

$$
\left\langle\uparrow _ { x } | _ { 1 } \left\langle\left.\uparrow_{z}\right|_{2} \quad \frac{1}{\sqrt{2}}\left(\left|\uparrow_{z}\right\rangle_{1}\left|\downarrow_{z}\right\rangle_{2}-\left|\downarrow_{z}\right\rangle_{1}\left|\uparrow_{z}\right\rangle_{2}\right) .\right.\right.
$$

We know that the following outcomes are obtained with certainty if only one of the measurements is performed: $\sigma_{z}^{(1)}=-1, \sigma_{x}^{(2)}=-1$, and $\sigma_{z}^{(1)} \sigma_{x}^{(2)}=-1$. This demonstrates the failure of the "product rule" for pre- and postselected quantum systems. If we consider the spatial dichotomic variable instead of the spin with the correspondence:

$$
\begin{aligned}
\left|\uparrow_{z}\right\rangle_{1} \equiv|A\rangle_{1}, \quad\left|\downarrow_{z}\right\rangle_{1} \equiv|B\rangle_{1}, \\
\left|\uparrow_{x}\right\rangle_{2} \equiv|A\rangle_{2}, \quad\left|\downarrow_{x}\right\rangle_{2} \equiv|B\rangle_{2},
\end{aligned}
$$

then again we reach the situation in which each particle is with certainty to be found in box $B$ if it is searched for there, but they cannot be found in $B$ together.

We can see another amusing feature of the two-state vector (18) when we recast it considering just one spin- $\frac{1}{2}$ particle. In this case we map the spin variable of the first EPR particle into the spatial variable of our particle via (19) and the spin of the second EPR particle becomes the spin of our particle. With this correspondence, the particle is pre-selected in the state

$$
\left|\Psi_{1}\right\rangle=\frac{1}{\sqrt{2}}\left(|A\rangle\left|\downarrow_{z}\right\rangle-|B\rangle\left|\uparrow_{z}\right\rangle\right)
$$

and post-selected in the state

$$
\left|\Psi_{2}\right\rangle=\frac{1}{\sqrt{2}}(|A\rangle+|B\rangle)\left|\uparrow_{z}\right\rangle .
$$

In this pre- and post-selected state the probability to find the particle in $A$ at intermediate times vanishes. The weak value $\left(\mathrm{P}_{\mathrm{A}}\right)_{w}$ vanishes so that, in some sense, the particle is not in $A$. Nevertheless, the weak measurement of the spin component $\sigma_{x}$ in $A$ yields $\left(\mathbf{P}_{\mathbf{A}} \sigma_{x}\right)_{w}=-1$. If the particle is an electron or a neutron, we will then sense a non-vanishing magnetic field in $A$ (if it is weakly measured), but the weak value of the number of particles in $A$ is zero. Thus, for "weak measurement reality" 27] the particle is in $B$, but its magnetic field is in $A$ which might be arbitrary far from $B$. For a possible interpretation of this result see [28].

In the above case, for weak coupling the particle is in $B$, but its magnetic field is in $A$. However, the particle generates magnetic field in $B$ too. By considering a particle in a generalized two-state vector [1], we can arrange that the magnetic field appears only in $A$. A particle is described by a generalized two-state vector $\sum_{i} \alpha_{i}\left\langle\Phi_{i}|\quad| \Psi_{i}\right\rangle$ when it and an ancilla (which nobody touches at the intermediate time) are pre- and postselected in particular entangled states. The weak value of an observable $O$ of the particle in this case is:

$$
O_{w}=\frac{\sum_{i} \alpha_{i}\left\langle\Phi_{i}|O| \Psi_{i}\right\rangle}{\sum_{i} \alpha_{i}\left\langle\Phi_{i} \mid \Psi_{i}\right\rangle}
$$

The generalized two-state vector which leads to the phenomena described above is:

$$
\begin{array}{r}
\left(\left\langleA | + \langle B | ) \left\langle\uparrow _ { z } \left|\quad\left(\left|\downarrow_{z}\right\rangle|A\rangle+\left|\uparrow_{z}\right\rangle|B\rangle\right)+\right.\right.\right.\right. \\
\left(\left\langleA | + \langle B | ) \left\langle\downarrow _ { z } \left|\quad\left(\left|\uparrow_{z}\right\rangle|A\rangle+\left|\downarrow_{z}\right\rangle|B\rangle\right) .\right.\right.\right.\right.
\end{array}
$$

Indeed, it is straightforward to see that

$$
\left(\mathbf{P}_{\mathbf{A}}\right)_{w}=0, \quad\left(\mathbf{P}_{\mathbf{B}}\right)_{w}=1
$$

Nevertheles, weak measurement can sense the particle's magnetic field only in $A$ :

$$
\begin{array}{r}
\left(\mathbf{P}_{\mathbf{A}} \sigma_{x}\right)_{w}=1, \quad\left(\mathbf{P}_{\mathbf{A}} \sigma_{y}\right)_{w}=\left(\mathbf{P}_{\mathbf{A}} \sigma_{z}\right)_{w}=0, \\
\left(\mathbf{P}_{\mathbf{B}} \sigma_{x}\right)_{w}=\left(\mathbf{P}_{\mathbf{B}} \sigma_{y}\right)_{w}=\left(\mathbf{P}_{\mathbf{B}} \sigma_{z}\right)_{w}=0
\end{array}
$$

i.e., there is a magnetic field along the $x$ axis corresponding to a particle in $A$ with spin $\sigma_{x}=1$.

We hope that the examples presented above will help to develop an intuition for understanding pre- and postselected systems with entanglement and will lead to useful applications of the peculiar effects such system exhibit.

We thank Eli Cohen for useful discussions.This work was supported in part by the Binational Science Foundation Grant 32/08 and the Israel Science Foundation Grant No. 1125/10. 
[1] Y. Aharonov and L. Vaidman, J. Phys. A 24, 2315 (1991).

[2] Y. Aharonov and L. Vaidman, Phys. Rev. A 67, 042107 (2003).

[3] K.J. Resch, J.S. Lundeen, and A.M. Steinberg, Phys. Lett A 324, 125 (2004).

[4] P. Kolenderski et al., Phys. Rev. A 86, 012321 (2012).

[5] R.E. George et al., arXiv:1205.2594 (2012).

[6] W. D. Sharp and N. Shanks, Phil. Sci. 60, 488 (1993).

[7] L. Vaidman, Stud. Hist. Phil. Mod. Phys. 30, 373 (1999).

[8] R. B. Griffiths, Phys. Rev. A 54, 2759 (1996); 57, 1604 (1998).

[9] A. Kent, Phys. Rev. Lett. 78, 2874 (1997).

[10] R. E. Kastner, Found. Phys. 29, 851 (1999).

[11] L. Vaidman, Found. Phys. 29, 865 (1999).

[12] A. Shimony, Erken. 45, 337 (1997).

[13] O. Cohen, Phys. Rev. A 51, 4373 (1995).

[14] L. Vaidman, Phys. Rev. A 57, 2251 (1998).

[15] R. E. Kastner, Stud. Hist. Phil. Mod. Phys. 35B, 57 (2004).
[16] K.A. Kirkpatrick, J. Phys. A 36, 4891 (2003).

[17] M. S. Leifer and R. W. Spekkens, Phys. Rev. Lett. 95, 200405 (2005).

[18] T. Ravon and L. Vaidman, J. Phys. A 40, 2873 (2007).

[19] K.A. Kirkpatrick, J. Phys. A 40, 2883 (2007).

[20] N. Aharon and L. Vaidman, Phys. Rev. A 77, 052310 (2008).

[21] Y. Aharonov and L. Vaidman, Phys. Rev. A 41, 11 (1990).

[22] O. Hosten, P. Kwiat, Science 319, 787-790 (2008).

[23] P.B Dixon, D. J. Starling, A. N. Jordan and J. C. Howell, Phys. Rev. Lett. 102, 173601 (2009).

[24] L. Hardy, Phys. Rev. Lett. 68, 2981 (1992).

[25] Y. Aharonov, A. Botero, S. Popescu, B. Reznik, and J. Tollaksen, Phys. Lett A 301130 (2002).

[26] L. Vaidman, Phys. Rev. Lett. 70, 3369 (1993).

[27] L. Vaidman, Found. Phys. 26, 895 (1996).

[28] Y. Aharonov, S. Popescu, and P. Skrzypczyk, arXiv:1202.0631 1 (2012). 